\title{
Atezolizumab compared to chemotherapy for first-line treatment in non-small cell lung cancer with high PD-L1 expression: a cost-effectiveness analysis from US and Chinese perspectives
}

\author{
Shuqiao Cheng ${ }^{1}$, Rui Pei ${ }^{1}$ Jianhuang $\mathrm{Li}^{2}$, Bin $\mathrm{Li}^{2}$, Lanhua Tang ${ }^{2}$, Tao Yin ${ }^{1}$, Shao Liu ${ }^{1}$ \\ ${ }^{1}$ Department of Pharmacy, Xiangya Hospital of Central South University, Changsha, China; ${ }^{2}$ Department of Oncology, Xiangya Hospital of Central \\ South University, Changsha, China \\ Contributions: (I) Conception and design: T Yin, S Liu; (II) Administrative support: T Yin, S Liu; (III) Provision of study materials or patients: J Li, \\ B Li, L Tang; (IV) Collection and assembly of data: S Cheng, R Pei; (V) Data analysis and interpretation: S Cheng; (VI) Manuscript writing: All \\ authors; (VII) Final approval of manuscript: All authors. \\ Correspondence to: Prof. Tao Yin, PhD; Prof. Shao Liu, PhD. Department of Pharmacy, Xiangya Hospital of Central South University, Xiangya Road \\ 87, Changsha 410008, China. Email: simon863@vip.sina.com; liushao999@csu.edu.cn.
}

\begin{abstract}
Background: The IMpower110 trial revealed that atezolizumab treatment had significantly longer overall survival (OS) than chemotherapy in non-small cell lung cancer (NSCLC) patients with high-programmed death ligand 1 (PD-L1) expression. The purpose of the present study was to estimate the cost-effectiveness of atezolizumab versus platinum-based chemotherapy for first-line treatment in metastatic NSCLC with high PD-L1 expression, from the perspective of US and Chinese payers.

Methods: A partitioned survival model was constructed based on information from the IMpower110 clinical trial to estimate cost-effectiveness of atezolizumab versus chemotherapy as first-line treatment of metastatic NSCLC. Costs were estimated from US and Chinese payer perspectives. The impact of uncertainty was explored by performing one-way and probabilistic sensitivity analyses.

Results: In the United States, treatment with atezolizumab was estimated to increase 0.87 quality adjusted life years (QALYs) at a cost of \$123,424/QALY. In China, the use of atezolizumab cost an additional \$68,489 compared with chemotherapy, yielding an incremental cost-effectiveness ratio (ICER) of \$78,936/QALY. Sensitivity analysis indicated that the cost of atezolizumab was the most influential factor in both countries.

Conclusions: In the United States, which had a willingness-to-pay (WTP) threshold of $\$ 100,000$ to \$150,000 per QALY, atezolizumab was a cost-effective strategy for first-line treatment in metastatic NSCLC patients with high PD-L1 expression when compared to chemotherapy. For China, with a WTP threshold of $\$ 33,210$ per QALY, atezolizumab was not considered good-value treatment for NSCLC, and a price reduction of $52 \%$ appeared to be justified.
\end{abstract}

Keywords: Cost-effectiveness; atezolizumab; non-small cell lung cancer (NSCLC); United States; China

Submitted Aug 02, 2021. Accepted for publication Sep 16, 2021.

doi: $10.21037 / \mathrm{atm}-21-4294$

View this article at: https://dx.doi.org/10.21037/atm-21-4294

\section{Introduction}

Lung cancer is the second most commonly diagnosed cancer and the leading cause of cancer-related deaths worldwide (1). Approximately $80 \%$ of all lung cancer cases are non-small cell lung cancer (NSCLC). A large proportion of patients are diagnosed at a metastatic stage (57\%), and their 5-year relative survival rate is only $6 \%$ (2).

Prior to the introduction of immune check-point inhibitors (ICIs), platinum-based chemotherapy was the first-line treatment for metastatic NSCLC patients without epidermal growth factor receptor (EGFR) or anaplastic lymphoma kinase (ALK) positive tumor mutations (3). 
In recent years, the emergence of ICIs has changed the treatment paradigm of metastatic NSCLC, and some ICIs, such as pembrolizumab, have already been widely used in clinical practice as a new standard of care (4).

Atezolizumab is an immunoglobulin G1 (IgG1) monoclonal antibody that binds to programmed death ligand 1 (PD-L1) on tumor cell surfaces and reactivates $\mathrm{T}$ cell antitumor functions by blocking interactions between PD-L1 and programmed death 1 (PD-1) (5). PD-L1 antibody was proved to induce durable tumor regression and prolonged stabilization of disease in NSCLC patients (6). The IMpower110 trial (7) demonstrated that atezolizumab treatment resulted in significantly longer OS than chemotherapy in NSCLC patients with high PD-L1 expression (PD-L1 expression on at least $50 \%$ of tumor cells or at least $10 \%$ of tumor-infiltrating immune cells as assessed), regardless of histologic type. Moreover, atezolizumab showed a manageable safety profile with a lower frequency of grade 3-4 treatment related adverse event (AE) than chemotherapy despite longer exposure. Based on this result, the US Food and Drug Administration (FDA) approved atezolizumab for first-line treatment of metastatic NSCLC with high PD-L1 expression (8). In China, the National Medical Products Administration (NMPA) approved the application of atezolizumab for firstline treatment of NSCLC on April 29, 2021 (9). These made atezolizumab the second ICI after pembrolizumab and first PD-L1 monotherapy in the treatment of high PD-L1 expression NSCLC.

Although the use of atezolizumab results in significant survival gains for NSCLC compared with chemotherapy, whether its cost reflects its potential benefit remains unknown. This study is the first economic investigation of atezolizumab for first-line treatment of NSCLC, from a US and Chinese perspective. The objective of our study was to evaluate the cost-effectiveness of atezolizumab versus platinum-based chemotherapy for first-line treatment in metastatic NSCLC with high PD-L1 expression. We present the following article in accordance with the CHEERS reporting checklist (available at https://dx.doi. org/10.21037/atm-21-4294).

\section{Methods}

We developed a partitioned survival model to evaluate the cost-effectiveness of using atezolizumab versus platinumbased chemotherapy in the first-line treatment of NSCLC. The outcomes were total cost, life-years (LYs), quality- adjusted LYs (QALYs), and incremental cost-effectiveness ratios (ICERs). A willingness-to-pay (WTP) threshold of $\$ 100,000$ to $\$ 150,000$ per QALY (10) and $\$ 33,210$ per QALY (3 times the gross domestic product per capita of China in 2020) were applied to the United States and China, respectively. A $3 \%$ annual discount rate was employed for both costs and outcomes (11). Statistical analysis was performed using $\mathrm{R}$ software (version 3.6.3, http://www. r-project.org).

\section{Population and interventions}

Medical information was derived from the IMpower110 trial (6). Patients with stage IV NSCLC who had not previously received chemotherapy were randomly assigned in a $1: 1$ ratio to receive either atezolizumab $(1,200 \mathrm{mg}$ intravenously, maximum 35 cycles) or platinum-based chemotherapy (once every 3 weeks, 4 or 6 cycles) (Table S1). Chemotherapy dosing was calculated for the model based on body surface area (United States, $1.8 \mathrm{~m}^{2}, 70 \mathrm{~kg}$; China, $1.72 \mathrm{~m}^{2}, 65 \mathrm{~kg}$ ) and creatinine clearance of $70 \mathrm{~mL} /$ minute $(12,13)$.

According to the IMpower110 trial, the proportion of patients who received at least 1 subsequent anticancer therapy was $29.6 \%$ in the atezolizumab arm and $49.5 \%$ in the chemotherapy arm. The distribution of subsequent therapies was based on data from the IMpower110 trial; subsequent therapy details are shown in Table S2. The duration of subsequent therapy was 2.7 months, based on a real-world retrospective study (14). Following treatment, patients received best supportive care until death. A 20-year time horizon was selected to accommodate patient life expectancy.

\section{Model structure}

The partitioned survival model was developed using TreeAge Pro 2021 software (TreeAge Software, Williamstown, Massachusetts, USA) with 3 mutually independent health states: progression-free survival (PFS), progressive disease (PD), and death (Figure 1). In this model, the proportion of patients in each health state at a certain time point was calculated based on the two survival curves of PFS and OS, with the advantages of incorporating the time-varying therapeutic effect naturally and no requirements for estimating transition probabilities (15).

PFS and OS curves for atezolizumab and chemotherapy treatment were based on the results of the IMpower110 


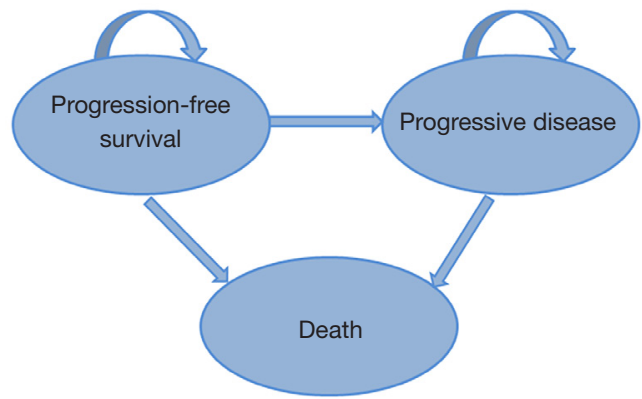

Figure 1 Model structure and transitions.

trial. Kaplan-Meier data were extracted by GetData Graph Digitizer (version 2.26; http://www.getdata-graph-digitizer. com), and time-to-event data were generated using the algorithm described in the study of Guyot et al. (16). Among the log-logistic, exponential, lognormal, generalized gamma, and Gompertz distributions, the best fit for the PFS and OS data of the atezolizumab arm was Weibull distribution, according to Akaike information criterion (AIC), Bayesian Information Criterion (BIC), and visual inspection. Log-logistic distribution fit the chemotherapy arm PFS and OS curves best, based on the same method (Figure S1).

\section{Cost and utility estimates}

Only direct medical care costs were covered in this model, including drug costs, administration costs, the cost of immunohistochemical testing, the cost of $\mathrm{AE}$ management, follow-up costs, best supportive care costs, and terminal care costs. Costs were estimated from US and Chinese payer perspectives. For the United States, drug costs were estimated using 2021 average sale pricing from the Centers for Medicare and Medicaid Services (17). Administration costs and follow-up costs were estimated based on the 2021 Medicare physician fee schedule (18). For China, all drug and administration costs were derived from Xiangya Hospital, Central South University and converted to US dollars with an exchange rate of $\$ 1=$ RMB 6.5443.

We included severe AEs (higher than grade 3) that occurred in more than $5 \%$ of the patients in the model (Table S3). For the United States, AE costs were derived from previously published studies $(19,20)$, and for China, they were calculated based on local charges. Best supportive care and terminal care costs were sourced from the literature (21-23). The Consumer Price Index was used to adjust costs for inflation to reflect 2021 US dollars. All information regarding costs is listed in Table 1.

Utility values were applied to reflect the impact of the disease on health states and were measured by patient preference for living at a particular health state, in which 0 represents the worst health and 1 the best. The utility data used in the model (Table 2) were obtained from previously published articles (24-27).

\section{Sensitivity analysis}

We conducted one-way sensitivity analysis to identify the key factors that influence cost-effectiveness. Variable ranges were obtained from the best available evidence; otherwise, a variance of $\pm 20 \%$ of base-case values was employed. Probabilistic sensitivity analysis was conducted by running 10,000 Monte Carlo simulations to test the uncertainty of the model with all parameters simultaneously varied within a specific pattern of distribution (Tables 1,2). As proposed by the Professional Society for Health Economics and Outcomes Research (ISPOR)-Society for Medical Decision Making (SMDM) Modeling Good Research Practices Task Force (28), gamma distribution was adopted for all input costs and beta distribution was used for probability and utility estimates (29).

\section{Results}

\section{Base-case analysis}

In the base case, atezolizumab showed an additional survival benefit of 1.27 life-years (LYs) compared to chemotherapy in high PD-L1 expression NSCLC patients. Accounting for quality of life, patients in the atezolizumab group survived 0.87 QALYs longer than the chemotherapy patients (1.75 vs. 0.88 QALYs). In the United States and China, the use of atezolizumab cost an additional $\$ 107,089$ and $\$ 68,489$, respectively, compared with chemotherapy, yielding an ICER of \$123,424/QALY and \$78,936/QALY, respectively (Table 3).

\section{Sensitivity analysis}

Figure 2 presents the results of one-way sensitivity analysis. Figure $2 A$ shows that for the United States, the variables with the greatest influence on the ICER were the cost of atezolizumab and utility for PFS of atezolizumab and of chemotherapy. The ICER exceeded the WTP threshold 
Table 1 Costs in the United States and China

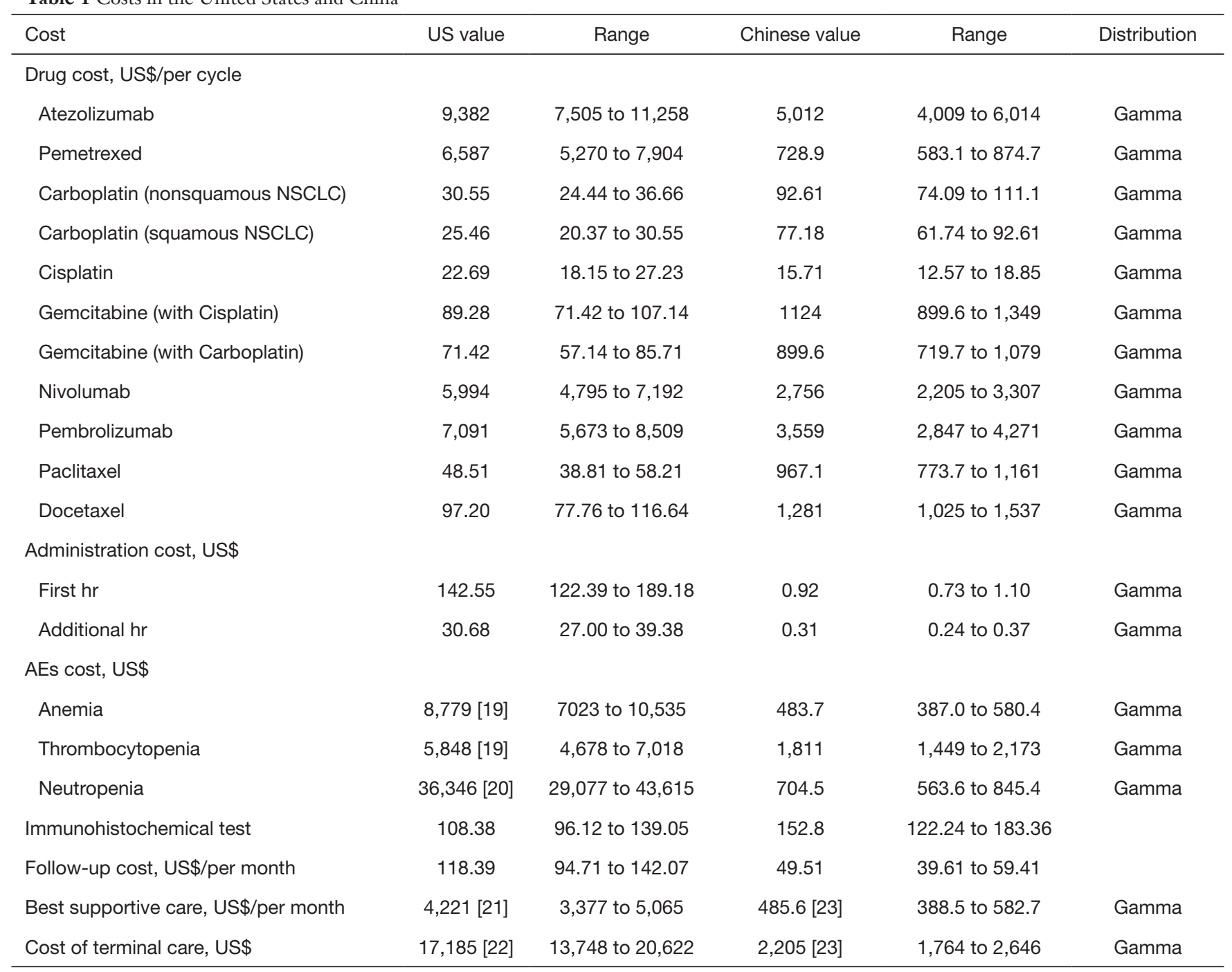

NSCLC, non-small cell lung cancer; AEs, adverse events.

of $\$ 150,000$ per QALY when the price of atezolizumab (\$7.82 per milligram) increased to the upper limit $(\$ 9.38$ per milligram) or the utility for PFS of atezolizumab (0.691) increased to 0.829. The ICER was lower than the WTP threshold of $\$ 100,000$ per QALY when the price of atezolizumab decreased to $\$ 6.25$ per milligram. For China (Figure 2B), the cost of atezolizumab, utility for PFS of atezolizumab, and the time horizon had considerable impact on the ICER. Across the variation ranges for all parameters, the ICERs remained greater than $\$ 60,000$ per QALY, which was higher than the Chinese WTP threshold.

For high PD-L1 expression patients in the United States, probabilistic sensitivity analysis indicated that when the
WTP was $\$ 100,000$ to $\$ 150,000$ per QALY, the probability of atezolizumab strategy being cost-effective compared with chemotherapy strategy was $13.1 \%$ and $85.8 \%$, respectively (Figure 3A). For China (Figure 3B), atezolizumab was not a cost-effective option compared to chemotherapy at the WTP threshold of $\$ 33,210$ per QALY. We found that when atezolizumab pricing decreased to $48 \%$ of its original cost, the ICER was below the WTP threshold.

\section{Discussion}

Almost one-quarter of all cancer deaths are due to lung cancer $(18.4 \%$ of total cancer deaths), making a significant 
Table 2 Key variables for model

\begin{tabular}{|c|c|c|c|c|c|}
\hline Variable & Atezolizumab arm & Range & Chemotherapy arm & Range & Distribution \\
\hline Anemia & 0.017 & 0.014 to 0.020 & 0.183 & 0.146 to 0.220 & Beta \\
\hline Thrombocytopenia & 0.003 & 0.002 to 0.004 & 0.072 & 0.058 to 0.086 & Beta \\
\hline Neutropenia & 0.007 & 0.006 to 0.008 & 0.175 & 0.140 to 0.210 & Beta \\
\hline Utility for PFS & 0.691 & 0.558 to 0.829 & 0.653 & 0.522 to 0.786 & Beta \\
\hline Utility for PD & 0.473 & 0.378 to 0.568 & 0.473 & 0.378 to 0.568 & Beta \\
\hline \multicolumn{6}{|l|}{ AEs disutility } \\
\hline Anemia (25) & -0.073 & -0.058 to -0.088 & -0.073 & -0.058 to -0.088 & Beta \\
\hline
\end{tabular}

AEs, adverse events; PFS, progression-free survival; PD, progressive disease.

Table 3 Base-case analyses in high PD-L1 expression population

\begin{tabular}{|c|c|c|c|c|c|c|}
\hline Results & \multicolumn{3}{|c|}{ United States } & \multicolumn{3}{|c|}{ China } \\
\hline LYs & 3.14 & 1.87 & 1.27 & 3.14 & 1.87 & 1.27 \\
\hline QALYs & 1.75 & 0.88 & 0.87 & 1.75 & 0.88 & 0.87 \\
\hline Total cost, \$ & 257,618 & 150,529 & 107,089 & 90,359 & 21,870 & 68,489 \\
\hline ICER, \$/QALY & & & 123,424 & & & 78,936 \\
\hline
\end{tabular}

PD-L1, programmed death ligand 1; ICER, incremental cost-effectiveness ratio; LY, life year; QALY, quality adjusted life year.

contribution to the rapidly growing global cost of cancer care (30). In recent years, ICIs have made a breakthrough in NSCLC treatment, but their high cost has placed great economic pressure on payers (31). Therefore, the association between clinical benefit and drug cost needs to be demonstrated to develop pricing strategies for immunotherapy drugs.

The phase 3 IMpower 110 trial showed that atezolizumab had significant survival benefits compared with chemotherapy for metastatic NSCLC patients with high PD-L1 expression. Our study aimed to evaluate the health and economic outcomes of atezolizumab as first-line treatment of metastatic NSCLC patients, from a US and Chinese perspective.

In the United States, the base-case ICER for atezolizumab of $\$ 123,424 /$ QALY fell within the acceptable
WTP threshold range of $\$ 100,000 /$ QALY $-\$ 150,000$ / QALY. Probabilistic sensitivity analysis showed that atezolizumab had a high probability $(85.8 \%)$ of being determined cost-effective over chemotherapy at the upper limit of WTP threshold. Recently, a cost-effectiveness analysis of atezolizumab compared with chemotherapy from US perspective was conducted (32), demonstrating that atezolizumab cost an additional $\$ 224,590$ (\$311,054 vs. $\$ 86,464)$ and provided survival gain of 1.32 QALYs $(2.36$ vs. 1.08 QALYs) compared with chemotherapy, yielding an ICER of \$170,730/QALY. These results have reached a conclusion that atezolizumab was estimated not to be cost-effective compared with chemotherapy for high PDL1expression NSCLC population which is inconsistent with ours. The main reason probably lies in different models used in the two studies; partitioned survival analysis 
A

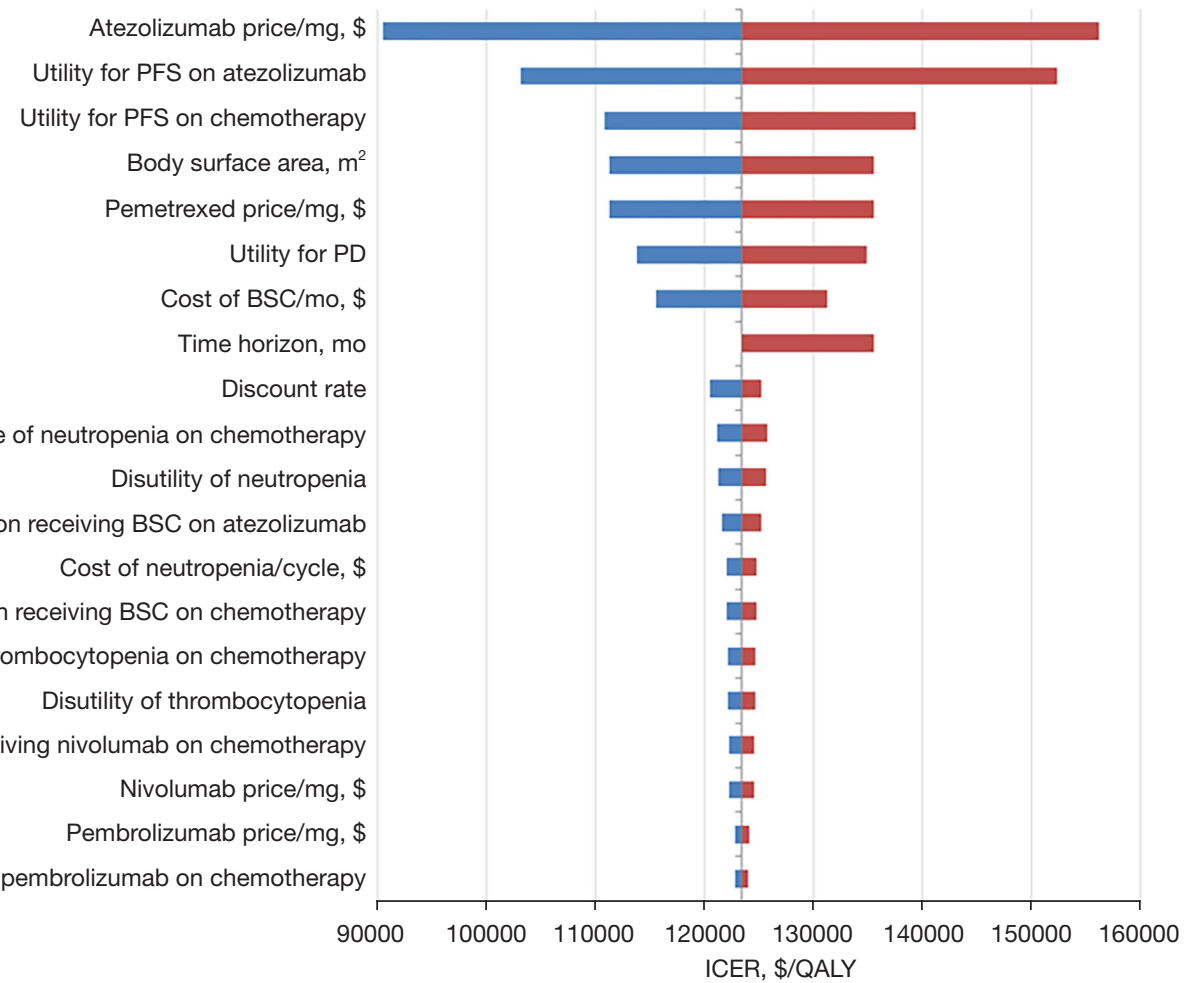

B

Atezolizumab price/mg, \$ Utility for PFS on atezolizumab

Time horizon, mo Utility for PFS on chemotherapy Utility for PD Discount rate

Body surface area, $\mathrm{m}^{2}$ Incidence of neutropenia on chemotherapy Disutility of neutropenia Pemetrexed price/mg, \$ Cost of BSC/mo, \$ Incidence of thrombocytopenia on chemotherapy Disutility of thrombocytopenia Proportion receiving nivolumab on chemotherapy Nivolumab price/mg, \$ Pembrolizumab price/mg, \$ Proportion receiving pembrolizumab on chemotherapy Incidence of anaemia on chemotherapy Disutility of anemia Proportion receiving BSC on atezolizumab

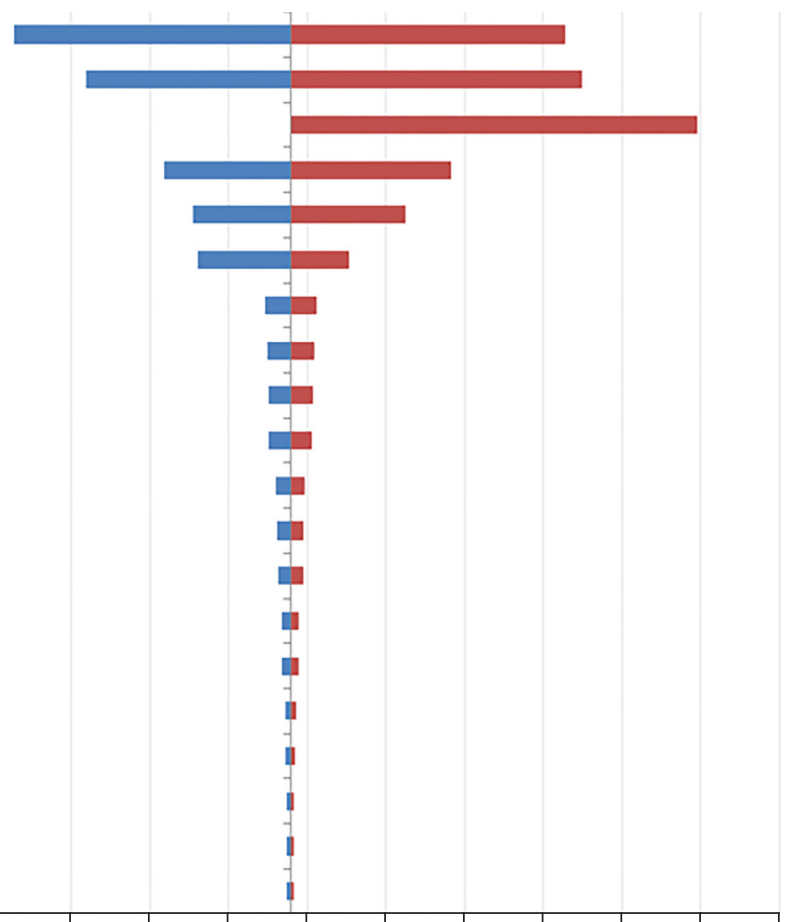
600006500070000750008000085000900009500010000105000110000 ICER, \$/QALY

Figure 2 Tornado diagrams of univariable sensitivity analyses. Results of ICER of atezolizumab versus chemotherapy for different model parameters of the United States (A) and China (B). The vertical black line intersecting the blue and red bars represents the ICER of \$123,424 and \$78,936 per QALY from the base case results. mg, milligram; PFS, progression free survival; PD, progressive disease; mo, month; BSC, best supportive care; ICER, incremental cost-effectiveness ratio; QALY, quality adjusted life year. 

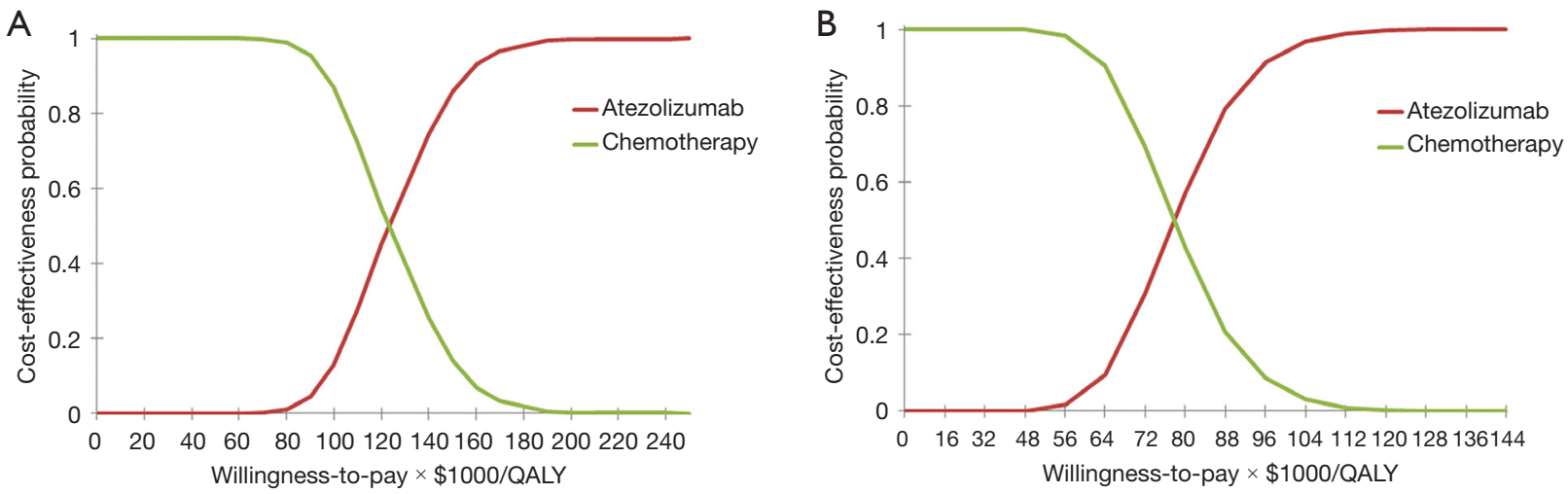

Figure 3 Cost-effectiveness acceptability curves. Results of probabilistic sensitivity analysis for atezolizumab versus chemotherapy for the United States (A) and China (B), which indicate the cost-effectiveness probability at different willingness-to-pay thresholds. QALY, quality adjusted life year.

model was designed in our study, while the published article developed a Markov model and calculated survival rate of atezolizumab by multiplying the survival rate of chemotherapy and the hazard ratios (HRs), resulting in longer QALY for atezolizumab group. Different variables and costs used for models may also explain the distinction between two ICERs. Before atezolizumab was approved by the FDA, pembrolizumab monotherapy was the standardof-care for metastatic nonsquamous NSCLC patients with PD-L1 tumor proportion score (TPS) $>50 \%$, and many studies have verified its cost-effectiveness compared to platinum-based chemotherapy in the United States $(18,23,33)$. Our results confirmed that after pembrolizumab, atezolizumab is the second immuno-monotherapy that has been proven to be cost-effective for people with high PDL1 expression, providing more treatment options for this population.

Until now, there has been no relevant study from a Chinese perspective for the economic evaluation of this therapeutic regimen. Contrary to the results of the United States, atezolizumab was not shown to be costeffective compared to chemotherapy in China, which had an ICER of $\$ 78,936$ per QALY, more than twice the WTP of $\$ 33,210$ per QALY. The results of one-way sensitivity analysis revealed the cost of atezolizumab was the most influential parameter, indicating that a price reduction may be a feasible strategy to increase the costeffectiveness of atezolizumab treatment. We found that when atezolizumab pricing decreased to $48 \%$ of its original cost, the ICER fell below the WTP threshold of $\$ 33,210$ per QALY, which resulted in atezolizumab becoming cost-effective. This finding could help with negotiating adjustments to the cost of atezolizumab to achieve more favorable economic results. Since 2017, the Chinese National Healthcare Security Administration has conducted price negotiations for oncology drugs, with the cost of some ICIs falling significantly in order to be included on the National Reimbursement Drug List (NRDL). The Chinese government has stated that future negotiations regarding oncology drug costs will be conducted on the basis of pharmacoeconomic evaluation, and thus our results could provide evidence for any future negotiations that may occur for atezolizumab. Based on the differing results from US and Chinese perspectives, it is evident that even for the same treatment, cost-effectiveness analysis outcomes from high-income and middle-income countries might be distinct. Thus, different regions should take into account locally representative economic parameters before drug approval.

Prior to the approval of atezolizumab monotherapy for first-line treatment of NSCLC, atezolizumab in combination with chemotherapy was shown to reduce overall mortality risk compared with chemotherapy alone. Several studies evaluated the cost-effectiveness of atezolizumab combination therapy, but the results indicated that the cost was not commensurate with the survival improvements it could provide. An analysis conducted by Criss et al. revealed that compared to treatment with bevacizumab, carboplatin, and paclitaxel (BCP), the addition of atezolizumab to $\mathrm{BCP}$ (ABCP) was estimated to obtain an ICER of \$201,676/QALY (34), while the results from another study indicated that the ICER of ABCP was 
\$568,967/QALY and \$516,114/QALY compared with BCP and carboplatin plus paclitaxel (CP), respectively (35). These results indicated that first-line treatment with $\mathrm{ABCP}$ was not a cost-effective treatment option for metastatic NSCLC in the United States. Cost-effectiveness analysis based on IMpower130 trial data have also shown that the addition of atezolizumab to carboplatin plus nab-paclitaxel is not an economically preferred treatment compared with chemotherapy for first-line treatment of NSCLC patients from a value standpoint in both the United States $(36,37)$ and China (38). Although atezolizumab combination therapy has clinical benefit, it is unlikely to be cost-effective for NSCLC patients compared to chemotherapy. Our study confirmed that atezolizumab monotherapy had the advantage of having a relatively lower total cost, making it cost-effective for NSCLC patients with high PD-L1 expression in the United States. Reducing the price of atezolizumab in China would be the most effective way to make this treatment strategy economical. In addition to the clinical benefit achieved in the treatment of NSCLC, atezolizumab has made a major breakthrough in extensivestage small-cell lung cancer (ES-SCLC). Atezolizumab or durvalumab combine with etoposide plus platinum chemotherapy (AEP and DEP respectively) significantly improve patients' survival and were approved by FDA as a first-line options for treating ES-SCLC. In a costeffectiveness and network meta-analysis study, AEP represented a dominant treatment strategy compared with DEP. Despite neither AEP nor DEP was cost-effective compared with EP chemotherapy, AEP was able to provide a more efficient balance between incremental cost and QALY than DEP (39). These achievements in advancedstage lung cancer inspired investigators to conduct further clinical trials in patients with early-stage lung cancer in both adjuvant and neoadjuvant therapies $(40,41)$, which call for corresponding cost-effectiveness research to elucidate the economic benefits of these therapies.

There were several limitations to our analysis. First, as we collected clinical information from the IMpower110 trial retrospectively, any biases within the trial could impact the outcomes of our model. Second, the median follow-up for high PD-L1 expression population in the IMpower110 clinical trial was 15.7 months, and because the model needed a time horizon long enough to simulate survival benefit and cost burden, we extrapolated the long-term PFS and OS curves using the short-term data of the clinical trial. When long-term survival data are available, we will perform a trial-based analysis to confirm or update current results. Third, we assumed that Chinese patients had the same utility values as US patients. Although this may lead to bias for the Chinese context, utility value variations were tested through sensitivity analysis, which showed that ICERs were always higher than the WTP within the settled range. Fourth, the cost of AEs of grade 1/2 or with a frequency lower than $5 \%$ in both treatment groups were excluded in this study. Some AEs, such as immune-related AEs, rarely occur but are costly to manage, which may lead to and underestimation of AE costs. Fifth, most basecase costs in China were derived from Xiangya Hospital, Central South University. Although these costs represent the price of medical care in most Chinese medical facilities, there may still be small differences across hospitals or regions, which could affect the generalizability of our findings.

\section{Conclusions}

In summary, for the United States, with a WTP threshold of $\$ 100,000$ to $\$ 150,000$ per QALY, atezolizumab was cost-effective for first-line treatment when compared with platinum-based chemotherapy in metastatic NSCLC patients with high PD-L1 expression. For China, atezolizumab was unlikely to be considered high-value treatment for NSCLC at a WTP threshold of $\$ 33,210$ per QALY, and a price reduction of $52 \%$ appeared to be justified.

\section{Acknowledgments}

Funding: None.

\section{Footnote}

Reporting Checklist: The authors have completed the CHEERS reporting checklist. Available at https://dx.doi. org/10.21037/atm-21-4294

Conflicts of Interest: All authors have completed the ICMJE uniform disclosure form (available at https://dx.doi. org/10.21037/atm-21-4294). The authors have no conflicts of interest to declare.

Ethical Statement: The authors are accountable for all aspects of the work in ensuring that questions related to the accuracy or integrity of any part of the work are appropriately investigated and resolved. 
Open Access Statement: This is an Open Access article distributed in accordance with the Creative Commons Attribution-NonCommercial-NoDerivs 4.0 International License (CC BY-NC-ND 4.0), which permits the noncommercial replication and distribution of the article with the strict proviso that no changes or edits are made and the original work is properly cited (including links to both the formal publication through the relevant DOI and the license). See: https://creativecommons.org/licenses/by-nc$\mathrm{nd} / 4.0 /$.

\section{References}

1. World Health Organization. Latest global cancer data: Cancer burden rises to 19.3 million new cases and 10.0 million cancer deaths in 2020. Date last accessed: March 2, 2021. Available online: https://www.iarc.fr/fr/newsevents/latest-global-cancer-data-cancer-burden-rises-to19-3-million-new-cases-and-10-0-million-cancer-deathsin-2020/

2. Siegel RL, Miller KD, Fuchs HE, et al. Cancer Statistics, 2021. CA Cancer J Clin 2021;71:7-33.

3. Zappa C, Mousa SA. Non-small cell lung cancer: current treatment and future advances. Transl Lung Cancer Res 2016;5:288-300.

4. Doroshow DB, Sanmamed MF, Hastings K, et al. Immunotherapy in Non-Small Cell Lung Cancer: Facts and Hopes. Clin Cancer Res 2019;25:4592-602.

5. Rittmeyer A, Barlesi F, Waterkamp D, et al. Atezolizumab versus docetaxel in patients with previously treated non-small-cell lung cancer (OAK): a phase 3, openlabel, multicentre randomised controlled trial. Lancet 2017;389:255-65.

6. Brahmer JR, Tykodi SS, Chow LQ, et al. Safety and activity of anti-PD-L1 antibody in patients with advanced cancer. N Engl J Med 2012;366:2455-65.

7. Herbst RS, Giaccone G, de Marinis F, et al. Atezolizumab for First-Line Treatment of PD-L1-Selected Patients with NSCLC. N Engl J Med 2020;383:1328-39.

8. US Food and Drug Administration. FDA approves atezolizumab for first-line treatment of metastatic NSCLC with high PD-L1 expression. Date last accessed: March 2, 2021. Available online:https://www.fda.gov/drugs/ resources-information-approved-drugs/fda-approvesatezolizumab-first-line-treatment-metastatic-nsclc-highpd-11-expression

9. National Medical Products Administration of China. Release of information on pending receipt of drug approval certificates. Date last accessed: May 2, 2021. Available online: https://www.nmpa.gov.cn/zwfw/sdxx/ sdxxyp/yppjfb/20210429162506168.html

10. Neumann PJ, Cohen JT, Weinstein MC. Updating costeffectiveness--the curious resilience of the $\$ 50,000$-perQALY threshold. N Engl J Med 2014;371:796-7.

11. Sanders GD, Neumann PJ, Basu A, et al.

Recommendations for Conduct, Methodological Practices, and Reporting of Cost-effectiveness Analyses: Second Panel on Cost-Effectiveness in Health and Medicine. JAMA 2016;316:1093-103.

12. Goulart B, Ramsey S. A trial-based assessment of the cost-utility of bevacizumab and chemotherapy versus chemotherapy alone for advanced non-small cell lung cancer. Value Health 2011;14:836-45.

13. Wu B, Chen H, Shen J, et al. Cost-effectiveness of adding rh-endostatin to first-line chemotherapy in patients with advanced non-small-cell lung cancer in China. Clin Ther 2011;33:1446-55.

14. Arunachalam A, Li H, Bittoni MA, et al. Real-World Treatment Patterns, Overall Survival, and Occurrence and Costs of Adverse Events Associated With SecondLine Therapies for Medicare Patients With Advanced Non-Small-Cell Lung Cancer. Clin Lung Cancer 2018;19:e783-99.

15. Chouaid C, Bensimon L, Clay E, et al. Costeffectiveness analysis of pembrolizumab versus standardof-care chemotherapy for first-line treatment of PD-L1 positive (>50\%) metastatic squamous and non-squamous non-small cell lung cancer in France. Lung Cancer 2019;127:44-52.

16. Guyot P, Ades AE, Ouwens MJ, et al. Enhanced secondary analysis of survival data: reconstructing the data from published Kaplan-Meier survival curves. BMC Med Res Methodol 2012;12:9.

17. Centers for Medicare and Medicaid Services. 2021 ASP Drug Pricing Files. Date last accessed: January 3, 2021. Available online: https://www.cms.gov/medicare/medicarepart-b-drug-average-sales-price/2021-asp-drug-pricingfiles

18. Centers for Medicare and Medicaid Services. License for Use of Current Procedural Terminology Fourth Edition (“СРТ®”). Date last accessed: January 3, 2021. Available online: https://www.cms.gov/apps/physician-fee-schedule/ search/search-results.aspx? $\mathrm{Y}=4 \& \mathrm{~T}=0 \& \mathrm{HT}=1 \& \mathrm{CT}=0 \& \mathrm{H} 1$ $=96415 \& \mathrm{H} 2=96413 \& \mathrm{H} 3=96417 \& \mathrm{M}=5$

19. Huang M, Lou Y, Pellissier J, et al. Cost Effectiveness of Pembrolizumab vs. Standard-of-Care Chemotherapy 


\section{Page 10 of 11}

as First-Line Treatment for Metastatic NSCLC that Expresses High Levels of PD-L1 in the United States. Pharmacoeconomics 2017;35:831-44.

20. Ting J, Tien Ho P, Xiang P, et al. Cost-Effectiveness and Value of Information of Erlotinib, Afatinib, and Cisplatin-Pemetrexed for First-Line Treatment of Advanced EGFR Mutation-Positive Non-SmallCell Lung Cancer in the United States. Value Health 2015;18:774-82.

21. Sheehan DF, Criss SD, Chen Y, et al. Lung cancer costs by treatment strategy and phase of care among patients enrolled in Medicare. Cancer Med 2019;8:94-103.

22. Huang M, Lopes GL, Insinga RP, et al. Costeffectiveness of pembrolizumab versus chemotherapy as first-line treatment in PD-L1-positive advanced nonsmall-cell lung cancer in the USA. Immunotherapy 2019;11:1463-78.

23. Lu S, Ye M, Ding L, et al. Cost-effectiveness of gefitinib, icotinib, and pemetrexed-based chemotherapy as firstline treatments for advanced non-small cell lung cancer in China. Oncotarget 2017;8:9996-10006.

24. She L, Hu H, Liao M, et al. Cost-effectiveness analysis of pembrolizumab versus chemotherapy as first-line treatment in locally advanced or metastatic non-small cell lung cancer with PD-L1 tumor proportion score $1 \%$ or greater. Lung Cancer 2019;138:88-94.

25. Nafees B, Stafford M, Gavriel S, et al. Health state utilities for non small cell lung cancer. Health Qual Life Outcomes 2008;6:84.

26. Handorf EA, McElligott S, Vachani A, et al. Cost effectiveness of personalized therapy for firstline treatment of stage IV and recurrent incurable adenocarcinoma of the lung. J Oncol Pract 2012;8:267-74.

27. Nafees B, Lloyd AJ, Dewilde S, et al. Health state utilities in non-small cell lung cancer: An international study. Asia Pac J Clin Oncol 2017;13:e195-203.

28. Briggs AH, Weinstein MC, Fenwick EA, et al. Model parameter estimation and uncertainty analysis: a report of the ISPOR-SMDM Modeling Good Research Practices Task Force Working Group-6. Med Decis Making 2012;32:722-32

29. Briggs AH, Claxton K, Sculpher MJ. Decision Modelling for Health Economic Evaluation. Oxford University Press, 2006.

30. Bray F, Ferlay J, Soerjomataram I, et al. Global cancer statistics 2018: GLOBOCAN estimates of incidence and mortality worldwide for 36 cancers in 185 countries. CA

\section{Cheng et al. Cost-effectiveness analysis of atezolizumab in NSCLC}

Cancer J Clin 2018;68:394-424.

31. Verma V, Sprave T, Haque W, et al. A systematic review of the cost and cost-effectiveness studies of immune checkpoint inhibitors. J Immunother Cancer 2018;6:128.

32. Peng Y, Zeng X, Peng L, et al. First-Line Atezolizumab for Metastatic NSCLC with High PD-L1 Expression: A United States-Based Cost-Effectiveness Analysis. Adv Ther 2021;38:2447-57.

33. Georgieva M, da Silveira Nogueira Lima JP, Aguiar P Jr, et al. Cost-effectiveness of pembrolizumab as first-line therapy for advanced non-small cell lung cancer. Lung Cancer 2018;124:248-54.

34. Criss SD, Mooradian MJ, Watson TR, et al. Costeffectiveness of Atezolizumab Combination Therapy for First-Line Treatment of Metastatic Nonsquamous NonSmall Cell Lung Cancer in the United States. JAMA Netw Open 2019;2:e1911952.

35. Wan X, Luo X, Tan C, et al. First-line atezolizumab in addition to bevacizumab plus chemotherapy for metastatic, nonsquamous non-small cell lung cancer: A United States-based cost-effectiveness analysis. Cancer 2019;125:3526-34.

36. Ding D, Hu H, Liao M, et al. Cost-Effectiveness Analysis of Atezolizumab Plus Chemotherapy in the First-Line Treatment of Metastatic Non-Squamous Non-Small Cell Lung Cancer. Adv Ther 2020;37:2116-26.

37. Lin S, Luo S, Zhong L, et al. Cost-effectiveness of atezolizumab plus chemotherapy for advanced non-smallcell lung cancer. Int J Clin Pharm 2020;42:1175-83.

38. Yang Z, Zhu Y, Xiang G, et al. First-line atezolizumab plus chemotherapy in advanced non-squamous non-small cell lung cancer: a cost-effectiveness analysis from China. Expert Rev Pharmacoecon Outcomes Res 2021. [Epub ahead of print]. doi: 10.1080/14737167.2021.1899813.

39. Liu Q, Luo X, Yi L, et al. First-Line ChemoImmunotherapy for Extensive-Stage Small-Cell Lung Cancer: A United States-Based Cost-Effectiveness Analysis. Front Oncol 2021;11:699781.

40. Shu CA, Gainor JF, Awad MM, et al. Neoadjuvant atezolizumab and chemotherapy in patients with resectable non-small-cell lung cancer: an open-label, multicentre, single-arm, phase 2 trial. Lancet Oncol 2020;21:786-95.

41. Wakelee HA, Altorki NK, Zhou CC, et al. IMpower010: Primary results of a phase III global study of atezolizumab 
versus best supportive care after adjuvant chemotherapy in resected stage IB-IIIA non-small cell lung cancer [EB/ OL]. ASCO 2021, abstract. Available online: https:// oncology.medicinematters.com/

(English Language Editor: A. Muijlwijk)

Cite this article as: Cheng S, Pei R, Li J, Li B, Tang L, Yin T, Liu S. Atezolizumab compared to chemotherapy for firstline treatment in non-small cell lung cancer with high PD-L1 expression: a cost-effectiveness analysis from US and Chinese perspectives. Ann Transl Med 2021;9(18):1481. doi: 10.21037/ atm-21-4294 


\section{Supplementary}

Table S1 Treatment regimens in IMpower110

\begin{tabular}{|c|c|c|c|c|c|c|c|}
\hline Regimen & $\begin{array}{l}\text { Histologic type } \\
\text { at diagnosis }\end{array}$ & Therapy & Dose & Frequency & Duration & Maintenance & Distribution \\
\hline $\begin{array}{l}\text { Atezolizumab } \\
\text { alone }\end{array}$ & - & Atezolizumab & $1,200 \mathrm{mg}$ & $\begin{array}{l}\text { Day } 1 \text { of every } \\
\text { 3-week cycle }\end{array}$ & $\begin{array}{c}\text { Maximum } 35 \\
\text { cycles }\end{array}$ & & \\
\hline \multirow{3}{*}{$\begin{array}{l}\text { Platinum- } \\
\text { based } \\
\text { chemotherapy }\end{array}$} & Nonsquamous & $\begin{array}{c}\text { Pemetrexed + } \\
\text { Cisplatin }\end{array}$ & $\begin{array}{l}500 \mathrm{mg} / \mathrm{m}^{2} \\
+75 \mathrm{mg} / \mathrm{m}^{2}\end{array}$ & $\begin{array}{l}\text { Day } 1 \text { of every } \\
\text { 3-week cycle }\end{array}$ & 4 or 6 cycles & Pemetrexed & $69.7 \%$ \\
\hline & \multirow[t]{2}{*}{ Squamous } & $\begin{array}{c}\text { Gemcitabine + } \\
\text { Cisplatin }\end{array}$ & $\begin{array}{l}1,250 \mathrm{mg} / \mathrm{m}^{2} \\
+75 \mathrm{mg} / \mathrm{m}^{2}\end{array}$ & $\begin{array}{l}\text { Gemcitabine Day } 1,8 \\
\text { and Cisplatin Day } 1 \text { of every } \\
\text { 3-week cycle }\end{array}$ & 4 or 6 cycles & \multirow[t]{2}{*}{$\begin{array}{l}\text { Best } \\
\text { supportive } \\
\text { care }\end{array}$} & \multirow[t]{2}{*}{$30.3 \%$} \\
\hline & & $\begin{array}{c}\text { Gemcitabine + } \\
\text { Carboplatin }\end{array}$ & $\begin{array}{c}1,000 \mathrm{mg} / \mathrm{m}^{2} \\
+ \text { AUC } 5\end{array}$ & $\begin{array}{c}\text { Gemcitabine Day } 1, \\
8 \text { and Carboplatin Day } 1 \\
\text { of every } 3 \text {-week cycle }\end{array}$ & 4 or 6 cycles & & \\
\hline
\end{tabular}

VAUC, area under the curve; IV, intravenous infusion.

Table S2 Summary of subsequent therapy

\begin{tabular}{|c|c|c|c|c|}
\hline Type of therapy & \multicolumn{2}{|c|}{ Atezolizumab alone } & \multicolumn{2}{|c|}{ Platinum-based chemotherapy } \\
\hline Anticancer therapy ${ }^{\#}$ & - & $29.6 \%$ & - & $49.5 \%$ \\
\hline \multirow[t]{5}{*}{ Chemotherapy } & Carboplatin & $17 \%$ & Carboplatin & $5.8 \%$ \\
\hline & Gemcitabine & $7.9 \%$ & Docetaxel & $12.3 \%$ \\
\hline & Pemetrexed & $10.1 \%$ & & \\
\hline & Cisplatin & $7.6 \%$ & & \\
\hline & Paclitaxel & $5.8 \%$ & & \\
\hline Immunotherapy & & & Nivolumab & $15.9 \%$ \\
\hline
\end{tabular}

\#, participants may have received more than 1 subsequent anticancer therapy and only the treatments with frequency greater than $5 \%$ were considered; *, the details of subsequent therapy were assumed based on data from IMpower110. 
A

Parametric survival analysis for PFS of atezolizumab and chemotherapy
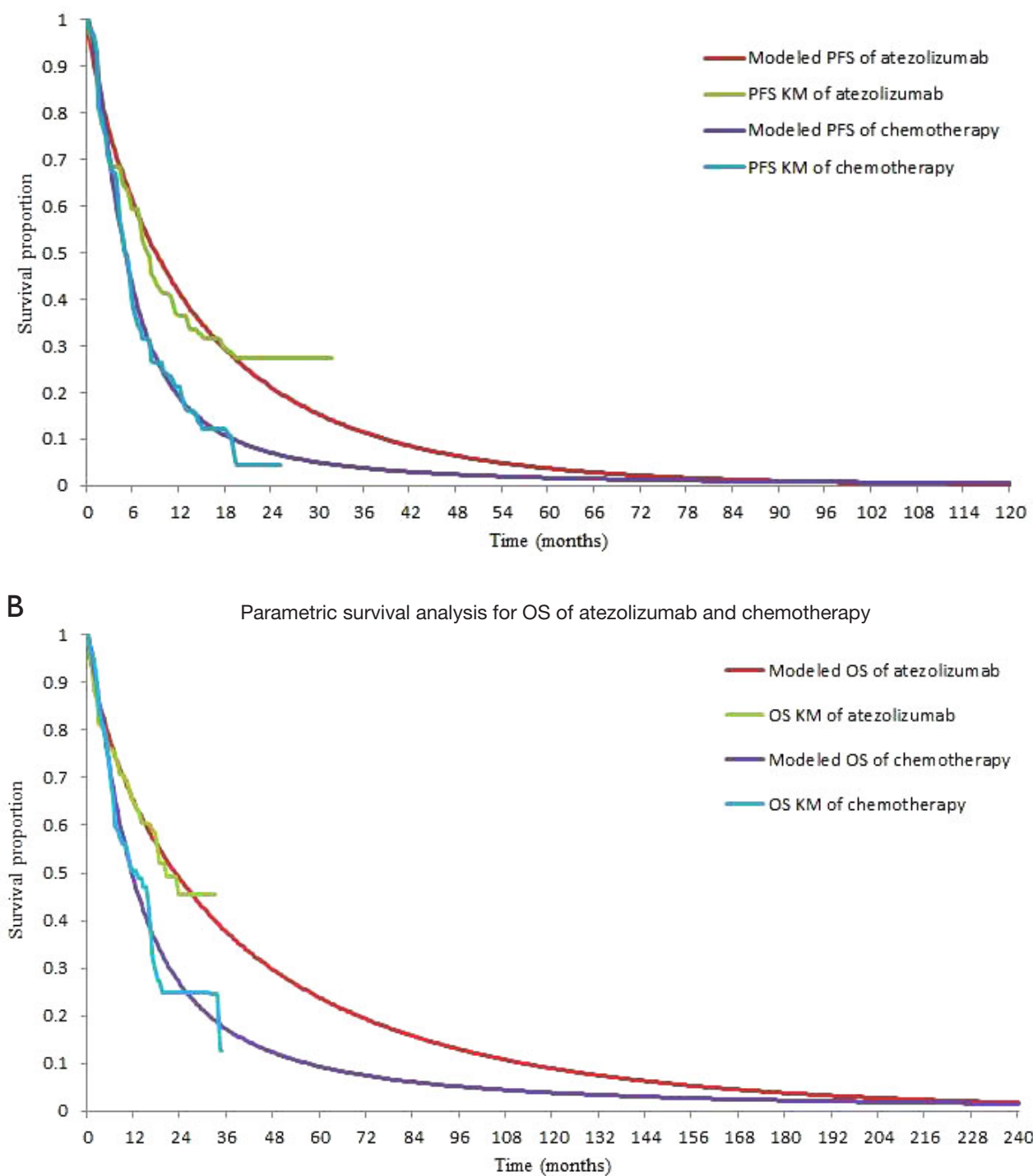

Figure S1 Parametric survival analysis. OS, overall survival; PFS, progression-free survival; KM, Kaplan-Meier.

Table S3 Grade 3-5 adverse events with a frequency greater than 5\% in IMpower110

\begin{tabular}{lcc}
\hline Adverse event & Atezolizumab alone, $\mathrm{n}(\%)$ & Platinum-based chemotherapy, $\mathrm{n}(\%)$ \\
\hline Anemia & $5(1.7)$ & $48(18.3)$ \\
Thrombocytopenia & $1(0.3)$ & $19(7.2)$ \\
Neutropenia & $2(0.7)$ & $46(17.5)$ \\
\hline
\end{tabular}

